\title{
Role of emotional intelligence on aggression: A comparison between adolescent boys and girls
}

\author{
Prangya Paramita Priyadarshini Das, Suchitra Tripathy \\ Department of Psychology, Ravenshaw University, Cuttack, Odisha, India
}

Email address:

prangya.ppdasresearch@gmail.com (P. P. P. Das), sweet.ctc1992@gmail.com (S. Tripathy)

To cite this article:

Prangya Paramita Priyadarshini Das, Suchitra Tripathy. Role of Emotional Intelligence on Aggression: A Comparison between Adolescent Boys and Girls. Psychology and Behavioral Sciences. Vol. 4, No. 1, 2015, pp. 29-35. doi: 10.11648/j.pbs.20150401.15

\begin{abstract}
Emotional intelligence (EI) is based on the concept of understanding one's own emotion and the emotions of others. Aggression is often categorized by hurtful and destructive behavior towards others; it seems to oppose the empathetic nature of EI. In this study the objective was to study the role of emotional intelligence on adolescents' aggression and also a gender difference in emotional intelligence and aggression of adolescents. The aggression scale developed by Buss \& Perry, in the year 1992 was used to measure aggression and the emotional intelligence scale developed by Anukool Hyde \& Sanjyo Pathe \& Upinder Dhar, in the year 1971 was used to measure emotional intelligence of adolescents. Data was collected from 64 adolescents from various Colleges and Universities. It was found that there exists a significant difference between the levels of emotional intelligences (HEI, MEI \& LEI) on aggression of adolescent girls as compared to adolescent boys. Boys were found to be more aggressive where as girls were found to be more emotionally intelligent. For boys emotional intelligence does not influence the level of aggression. There could be some other factors (peer pressure, media violence, and influence of drug) which play an important role in the development of aggression.
\end{abstract}

Keywords: Emotional Intelligence, Adolescence, Aggression

\section{Introduction}

Besides the ability of people to receive external stimuli through the five senses, human bodies respond internally by receiving and understanding information through affective states like emotions and feelings. The concept of emotional intelligence proposes that intelligence may understand emotion, and that emotion may facilitate intelligence (Mayer \& Ciarrochi, 2006). According to Akinboye (2002) "no human action, whether good or bad, is emotion free". Emotional intelligence is one of the important variables and has greater influence on human character. It has a pivotal role in the success of various domain of life. The basic emotions are happiness, interest, surprise, fear, anger, sorrow and disgust. According to Leventhal (1982) each of these emotions are operating through a control mechanism which serve as a monitor for one of the main aspects of human life. Controlling one's emotions does not happen automatically. This can be done when someone is in touch with his inner as well as outer world.

Each individual expresses emotion differently and ineffective expression of emotion creates abnormality within the person. Mental health is related with adequate expression of emotion. Adequate expression of emotion is related with the ability to understand, perceive and control one's and other's emotion. Aggression is directly linked with emotion and it determines one's behavior, personality and integrity. It determines the nature of an individual. Emotional intelligence is related with empathy (Ciarrochi, Chan, and Caputi, 2000). Research evidence shows that there exist a significant negative correlation between emotional intelligence and aggression.

Emotional Intelligence among adolescents affects adolescents' level of interpersonal relationship. Low level of emotional intelligence among adolescents leads to adolescent substance and illegal drug abuse, poor interpersonal relationship, high level of aggression, and physical fights (Brackett, Mayer and Warner, 2004). Emotional Intelligence plays a pivotal role in all realm of life. Keeping in view the significance of emotional intelligence on adolescents' level of aggression and gender difference the following objectives were formulated. 


\subsection{Objectives}

1 To study the role of emotional intelligence on adolescents' aggression.

2 To study the gender difference in adolescents' aggression with reference to the levels of emotional intelligence (high emotional intelligence, medium emotional intelligence and low emotional intelligence).

\section{Method}

\subsection{Sample}

The size of the sample was 64. Data was collected from 64 adolescent students from UG to PG level. Out of 64 students 30 were male and 34 were female adolescents. The age range was in between 18-24 years. All students were belonged to middle socioeconomic status.

\subsection{Tools}

Two questionnaires were used in this study. One was emotional intelligence scale and other one was aggression scale.

The emotional intelligence scale (EIS) developed by Anukool Hyde and Sanjyoi Pathe and Upinder Dhar in the year 1971. It was a 5 point scale. This scale was designed to measure Emotional Intelligence. This scale consisted of 34 items and the highest score is 170 and lowest score is 34 . The scale measures 10 factors dimensions, such as self awareness, empathy, self motivation, emotional stability, meaning relation, integrity, self development, value orientation, commitment and altruistic behavior. The reliability of the scale was 0.88 and the validity of the scale was 0.93 . Each item of the scale would be scored 5 for strongly agree, 4 for agree, 3 for neutral, 2 for disagree, and 1 for strongly disagree.

The aggression scale developed by Buss \& Perry, in the year 1992 was used in this study. It was a 5 point scale. This scale was designed to measure aggression. This scale consisted of 29 items and the highest score is 145 and the lowest score is 29. This scale consists of 4 factors, such as Physical Aggression (PA), Verbal aggression (VA), Anger (A), Hostility $(\mathrm{H})$. The reliability of this scale is 0.60 and the validity of this scale is 0.84 . Each item of this scale would be scored as 5 for extremely characteristic of me, 4 for somewhat characteristic of me, 3 for neither uncharacteristic nor characteristic of me, 2 for somewhat uncharacteristic of me and 1 for extremely uncharacteristic of me.

\subsection{Procedure}

The present study was conducted on adolescent students within the age bracket of 18 to 24 years. The scales were used to study the role of emotional intelligence on aggression of adolescent students. The instructions of these questionnaires were clearly explained to the subjects and care was taken to ensure that they understood the items in both the scale. All subjects were asked to clarify their doubts before answering the questionnaires and instructed to give a tick mark for each item in emotional intelligence scale and for aggression questionnaire subject put one of the numbers from $1,2,3,4$, and 5 , for each item according to his or her choice. The sample consisted of 30 males and 30 females. Each subject gave his or her responses for both scales. They were asked to respond to each item in the questionnaires freely without any hesitation. All subjects individually administered their questionnaires and were asked to complete the questionnaire without any time limit. Subjects were very happy at the time of data collection when they got to know about their level of aggression and emotional intelligence. This study was conducted in compliance with "ethical" principles.

\section{Results and Interpretation}

Table I. Descriptive Statistics for Adolescent Boys.

\begin{tabular}{llll}
\hline & N & Mean & SD \\
\hline High Emotional Intelligence (HEI) & 12 & 86.333 & 6.879 \\
Medium Emotional Intelligence (MEI) & 12 & 92.083 & 7.204 \\
Low Emotional Intelligence (LEI) & 6 & 83.833 & 11.321 \\
Total & 30 & 88.133 & 8.447 \\
\hline
\end{tabular}

Dependent Variable: Aggression

From Table-I it was found that out of 30 adolescent boys 12 belongs to HEI, 12 belongs to MEI and 6 belongs to LEI respectively. The mean score of aggression with high emotional intelligence is 86.33 , the mean score of aggression with medium emotional intelligence is 92.08 and the mean score of aggression with low emotional intelligence is 83.83 respectively. One-way analysis of variance (ANOVA) was applied to know the significance of mean difference between the three groups (HEI, MEI \& LEI).

Table II. One-Way ANOVA showing the role of EI on Aggression among Boys.

\begin{tabular}{llllll}
\hline & $\begin{array}{l}\text { Sum of } \\
\text { Squares }\end{array}$ & df & $\begin{array}{l}\text { Mean } \\
\text { Square }\end{array}$ & F & Sig. \\
\hline Between group & 337.050 & 2 & 168.525 & & \\
Within group & 1732.417 & 27 & 64.164 & 2.626 & .091 \\
Total & 2069.467 & 29 & & & \\
\hline
\end{tabular}

Dependent Variable: Aggression

The findings of one-way ANOVA shown in Table-II revealed that the effect of emotional intelligence on aggression for adolescent boys is not significant $\mathrm{F}(2,27)=$ $2.626, p=.091$. So there is no significant difference between adolescents boys with HEI, MEI and LEI respectively with regard to their aggression score.

Table III. Descriptive Statistics for Adolescent Girls.

\begin{tabular}{llll}
\hline & N & Mean & SD \\
\hline High Emotional Intelligence (HEI) & 17 & 66.235 & 4.493 \\
Medium Emotional Intelligence (MEI) & 12 & 72.583 & 10.448 \\
Low Emotional Intelligence (LEI) & 5 & 92.800 & 3.114 \\
Total & 34 & 72.382 & 11.401 \\
\hline
\end{tabular}

Dependent Variable: Aggression 
From Table-III it was found that out of 34 adolescent girls 17 belongs to HEI, 12 belongs to MEI and 5 belongs to LEI respectively. The mean score of aggression with high emotional intelligence is 66.23 , the mean score of aggression with medium emotional intelligence is 72.58 and the mean score of aggression with low emotional intelligence is 92.80 respectively. One-way analysis of variance (ANOVA) was applied to know the significance of mean difference between three groups (HEI, MEI \& LEI).

Table IV. One-Way ANOVA showing the role of EI on Aggression among Girls.

\begin{tabular}{llllll}
\hline & $\begin{array}{l}\text { Sum of } \\
\text { Squares }\end{array}$ & df & $\begin{array}{l}\text { Mean } \\
\text { Square }\end{array}$ & F & Sig \\
\hline Between group & 2727.254 & 2 & 1363.627 & & \\
Within group & 1562.775 & 31 & 50.412 & 27.050 & .000 \\
Total & 4290.029 & 33 & & & \\
\hline
\end{tabular}

Dependent Variable: Aggression

The findings of one-way ANOVA shown in Table-IV revealed that the effect of emotional intelligence on Aggression for adolescent girls is significant $F(2,27)=$ 27.050, $\mathrm{p}=.000$. So there exists a significant difference among adolescent girls with HEI, MEI and LEI respectively with regard to their aggression score.

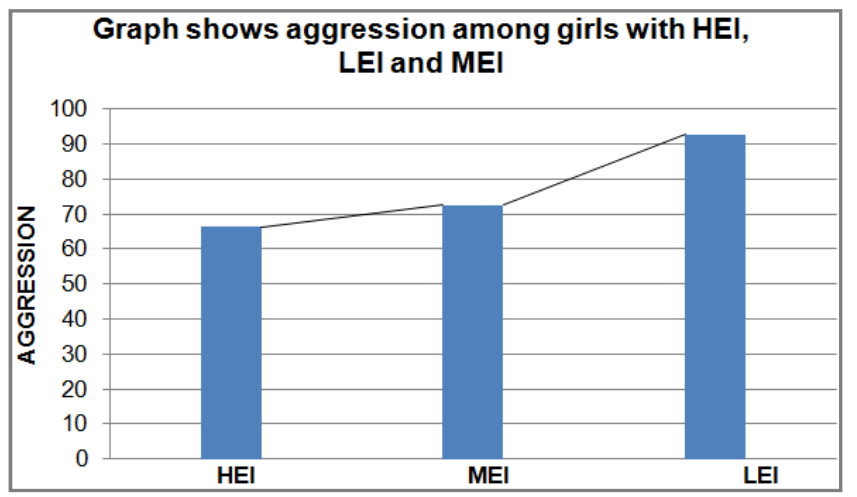

Figure I. Bar diagram showing aggression among girls with HEI, MEI and LEI.

The above figure indicates that girls with HEI showed low aggression as compared to girls with MEI and LEI.

Now that we have rejected the null hypothesis, it is appropriate to perform a Post-Hoc test to discover whether the three groups (HEI, MEI and LEI) are different. Scheffe test was applied since it has the distinction of being one of the safest of all possible post hoc tests.

Table V. Multiple Comparisons-Scheffe test.

\begin{tabular}{llll}
\hline (I) IV levels & $(\mathbf{J})$ IV Levels & Mean difference $(\mathbf{I}-\mathbf{J})$ & Sig \\
\hline \multirow{2}{*}{ HEI } & MEI & -6.34 & .075 \\
& LEI & $-26.56\left(^{*}\right)$ & .000 \\
\multirow{2}{*}{ MEI } & HEI & 6.34 & .075 \\
& LEI & $-20.21\left(^{*}\right)$ & .000 \\
LEI & HEI & $26.56(*)$ & .000 \\
& MEI & $20.21(*)$ & .000 \\
\hline
\end{tabular}

Dependent Variable: Aggression

*The mean difference is significant at the .05 level.
It was found from the above table that the mean difference between HEI and LEI is significant $(\mathrm{I}-\mathrm{J}=-26.56, \mathrm{p}=.000)$ and the mean difference between MEI and LEI is also significant $(\mathrm{I}-\mathrm{J}=20.21, \mathrm{p}=.000)$. But the mean difference between HEI and MEI is not significant $(I-J=6.34, p=.075)$.

Table VI. T-test showing the differences in emotional intelligence among adolescent boys and girls.

\begin{tabular}{llllllll}
\hline & Groups & N & Mean & SD & T & df & Sig \\
\hline Emotional & Boys & 30 & 76.66 & 22.40 & \multirow{2}{*}{2.03} & \multirow{2}{*}{62} & \multirow{2}{*}{.046} \\
Intelligence & Girls & 34 & 87.02 & 18.22 & & & \\
\hline
\end{tabular}

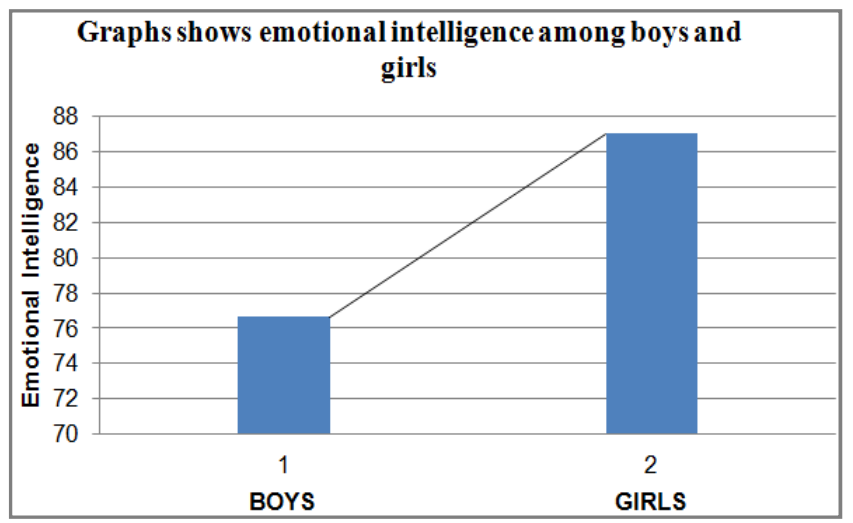

Figure II. Bar diagram showing emotional intelligence among boys and girls.

The above figure indicates that adolescent girls are more emotionally intelligent as compared to adolescent boys.

Table VII. T-test showing the differences in aggression among adolescent boys and girls.

\begin{tabular}{llllllll}
\hline & Groups & N & Mean & SD & t & df & Sig \\
\hline \multirow{2}{*}{ Aggression } & Boys & 30 & 88.13 & 8.44 & \multirow{2}{*}{6.209} & \multirow{2}{*}{62} & \multirow{2}{*}{.000} \\
& Girls & 34 & 72.38 & 11.40 & & \\
\hline
\end{tabular}

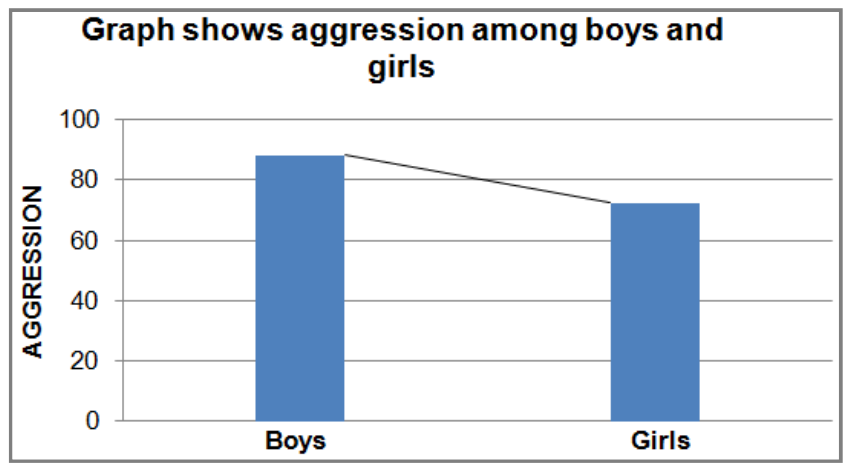

Figure III. Bar diagram showing aggression among boys and girls.

The above figure indicates that adolescent boys are more aggressive as compared to adolescent girls.

\section{Discussion}

An effort was completed in this piece of research to gain insights into the statistically significant differences among adolescents of high, medium and low emotional intelligence 
with regard to their aggression scores.

\subsection{Major Findings}

- There exists no significant difference among boys with high, medium and low emotional intelligences with regard to their aggression scores.

- There exists a significant difference among girls of high, medium and low emotional intelligences with regard to their aggression scores.

- The mean difference between the group HEI and LEI was significant for adolescent girls with regard to their aggression score.

- The mean difference between the group MEI and LEI was significant for adolescent girls with regard to their aggression score.

- There was no statistical significant difference between HEI and MEI for adolescent girls with regard to their aggression score.

- Girls were found to be more emotionally intelligent as compared to boys.

- Boys were found to be more aggressive as compared to girls.

From this piece of research we can say that emotional intelligence does not affect aggression among boys. ANOVA table for boys indicates that for boys there is no significant difference between HEI, MEI \& LEI with regard to aggression score. So, emotional intelligence is not a factor for aggression among adolescent boys. There were some other factors which actually affect the score of aggression among adolescent boys. According to National Youth Violence Prevention Resource Centre, USA, there are certain risk factors which increase the likelihood of aggression among adolescent boys.

\subsection{Individual Characteristics}

A number of individual characteristics have been shown to increase a child's risk for aggressive behavior particularly among boys. These include a difficult temperament as an infant, low intelligence, hyperactivity, impulsivity, and attention problems (Pepler \& Slaby, 1994). Additionally, aggressive boys frequently have poor social problem-solving skills: they often misinterpret other children's behavior as hostile, and they are often unable to find non-aggressive solutions to conflicts (Dodge, 1991).

\subsection{Home Environment}

Some characteristics of the home environment can increase the risk that a child will eventually become involved in aggressive behavior. Children and teens who come from homes where parents are coercive or manipulative with their children, provide little emotional support, do not monitor their activities, or have little involvement in their lives are at greater risk for engaging in aggressive behavior. Additionally, the use of harsh punishments or inconsistent discipline has been shown to be related to aggressive behavior in youth (Pepler \& Slaby, 1994).

\subsection{Relationships with Peers}

Because of their aggressive behavior and lack of social skills, highly aggressive adolescent boys are often rejected by their peers (Coie \& Dodge, 1990). This early rejection is predictive of later aggressive and violent behavior (USDHHS, 2001). However, by the teen years, most aggressive youth are not friendless, but have developed friendships with other teens with antisocial attitudes and behavior (Cairna \& Nickerman, 1998). Friendships with antisocial peers can be an important predictor of aggressive behavior and violence in the teenage years (USDHHS, 2001).

\subsection{School Failure}

Early behavior problems often lead to poor school achievement and school failure, which are important risk factors for delinquency and involvement with antisocial peer groups in the teenage years (Hawkins, Cleve \& Catalano, 1996).

\subsection{Exposure to Media Violence}

There is now solid evidence to suggest a relationship between aggressive behavior and exposure to violent television and movies (USDHHS, 2001). Researchers have found that adolescent boys are more physically and verbally aggressive immediately after watching violent television and movies. It is also clear that aggressive children and teens watch more violent television than their less aggressive peers.

\subsection{Community and Societal Factors}

Poverty, joblessness, discrimination, and societal acceptance of aggression all increase the risk of aggressive behavior (Hann \& Borek, 2002). Neighborhood factors that increase the likelihood that children and teens will become aggressive and involved in other problem behaviors include exposure to violence, the availability of drugs, alcohol and firearms, extreme poverty, neighborhood disintegration (e.g., vacant lots, graffiti, crime, drug dealing, and boarded-up houses, and resident views that are tolerant of misbehavior and violence) (Hewell, 1995).

ANOVA table for girls indicates that there exists a significant difference between HEI, MEI and LEI with regard to aggression scores. The significant $\mathrm{F}$ ratio indicates that emotional intelligence has a significant effect upon the aggression of adolescent girls. Post hoc comparisons using scheffe test indicated that the mean difference between MEI and LEI was significant which suggests that girls with MEI shows low level of aggression as compared to girls with LEI. The mean difference between LEI and HEI was significant which indicates that girls with LEI show high level of aggression as compared to LEI. So, low emotional intelligence is associated with high level of aggression. However it should be noted that emotional intelligence level should be low in order to see an effect. High emotional intelligence levels do not appear to significantly increase the level of aggression among girls. 
In fact, $80 \%$ of life successes in a person's life can be attributed to emotional intelligence (Goleman, 1995). High emotional intelligence levels have been correlated with a myriad of benefits and positive life outcomes. Advantages include high levels of happiness, health, well-being, better academic performance and an increased ability to cope with change (Qualter et al., 2007; Salami, 2011). This is not just true of adults; adolescents who have more advanced emotional abilities show low stress, fewer signs of aggression, and demonstrate a smaller likelihood of involvement with drugs and alcohol (Qualter et al., 2007). Abraham (1999) contended that those individuals who have higher levels of emotional intelligence have a stronger ability to empathize, generally leading to their ability to conform better to organizational requirements.

Just as high levels of emotional intelligence are beneficial, low levels create recognizable deficits. Importantly, however, the problems caused by low levels of EI are not just the absence of those beneficial traits exemplified by those with high EI. Low levels of EI have been correlated with some forms of mental illness, including depression, aggression, borderline personality disorder and difficulties in processing emotional information (Grewal \& Salovey, 2006). Those with low EI levels also tend to have a hard time understanding situations from the perspective of others and therefore tend to be less empathetic (Henley \& Long, 1999).

Siaruchi and his colleagues selected 73 boys and 58 girls of 13 to 18 age group to whom Schuttle's Emotional Intelligence Scale was given. The girls were meaningfully higher than the boys in overall emotional intelligence, emotional understanding, emotional regulation and emotional utilization at $\mathrm{P}<0.05$ level.

The fact that males are more aggressive than females has to do with biological, psychological, and social factors. Research shows in addition that males are more likely than females to behave in an aggressive manner. There is evidence that differences in socialization, cognition and personality may help explain gender-based disparity in rates of antisocial, as well as violent behaviors. Gender differences in cognition, socialization, and behavior may exist as early as infancy. This is when boys are able to express themselves at a higher rate. Infant girls show greater control over their emotions, whereas boys are more easily angered and depend more on inputs from their mother's. Psychologist shows that the ways which females and males are socialized affect their development. Males learn to value independence, while females are taught that their self-worth depends on their ability to maintain relationships. Although there are a few differences in aggression during the first few years of life, girls are socialized to be less aggressive than boys are. In connection girls are supervised more closely than boys are. Males are more likely to display physical aggression, while females display relational aggression (Welsh, 2007).

Low trait EQ may be a key ingredient in a variety of deviant behavior, many of which have been repeatedly linked to emotional deficits among adolescents (Bracket, Warner \& Mayer, 2004). Emotional competence is a complex phenomenon consisting of number of distinct, yet interrelated component skills including emotional appraisal, emotional expression and emotional understanding. A study undertaken by Bohnert, Crnic and Lim (2003) suggests that adolescents with aggressive behavior more often make errors interpreting intent in ambiguous social situations.

\subsection{Limitations}

The study is related with only one factor that is emotional intelligence. So attempt should be made to know the role of other factors (home environment, media violence, peer pressure and school failures etc.) on adolescent aggression.

\subsection{Implications}

Socialization process plays a very important role in the development of emotional intelligence among adolescents in general and boys in particular. It is the responsibility of the parents; teachers and the community to play an active role in the socialization process of adolescents to nurture the trait of emotional intelligence and decreasing the level of aggression. Among boys emotional intelligence is not an important factor that affects aggression. For boys specifically there are some vulnerable factors that affect the score of aggression which needs to be taken care of in an early stage, so that it would not affect the overall personality development and life of an adolescent.

\subsection{Direction for Further Research}

A recommendation is made to conduct a research on understanding aggression among adolescent by taking some more predictor variables like self concept, influence of drug addiction and peer pressure. Age differences among adolescents (early adolescence, middle adolescence and late adolescence) can also be taken into consideration while addressing the issue of aggression.

\section{Conclusion}

While decisive conclusions may be premature, the current investigation lends support to the notion that low level of intelligence is related with high level of aggression among adolescent girls. Boys were more aggressive whereas girls were found to be more emotionally intelligent. What makes this research so valuable is the unique ability of emotional intelligence to be taught. Individuals who have a higher emotional intelligence would be more able to conform to preconceived societal norms, therefore being less aggressive and less likely to break the law.

\section{Acknowledgement}

We are thankful to 64 adolescents without whom these data would not be available. 


\section{Contribution of Author}

The authors certify that both the authors have participated sufficiently in the intellectual content, conception and design of this work and the analysis and interpretation of the data as well as writing of the manuscript. The authors have been involved in drafting the manuscript for important intellectual content and have given final approval of the version to be published.

\section{References}

[1] Akinboye, J. O. (2002). Creativity Innovation and Success.Ibadan: Stirling-Horden Publishers (Nig.) Ltd. P. 32.

[2] Akinboye, J. O. (2002). Creativity Innovation and Success.In M. S. Eniola, (2007). The Influence of Emotional Intelligence and Self-Regulation Strategies on Remediation of Aggressive Behaviours in Adolescent with Visual Impairment. EthnoMed., 1(1), 71- 77

[3] Bernet, M. (1996).Emotional intelligence: Components and correlates. Paper presented at the 104th Annual Meeting of the American Psychological Association. Chicago.

[4] Brackett, M.A., Warner, R.M. \& Mayer, J.D. (2004).Emotional intelligence and its relation to everyday behaviour. Personality and Individual Differences, 36(6): 1387-1402.

[5] Ciarrochi, J., Dean, F.P., Wilson, C.J. \&Rickwood, D. (2002). Adolescents who need help the most are the least likely to seek it: the relationship between low emotional competence and low intention to seek help. British Journal of Guidance \& Counselling, 30(2):173-188.

[6] Ciarrochi, J. V., Chan, A.Y. C. \&Caputi, P. (2000).A critical evaluation of the emotional intelligence construct. Personality and Individual Differences, 28(3), 539-561.

[7] Card, N. A., Stucky, B. D., Sawalani, G. M., \& Little, T. D. (2008). Direct and indirect aggression during childhood and adolescence: A meta-analytic review of gender differences, intercorrelations, and relations to maladjustment. Child Development, 79(5), 1185-1229. doi:10.1111/j.14678624.2008.01184.x

[8] Cooper, R. K. and A. Sawaf. (1997). Executive Emotional Intelligence in Leadership and Organization. New York: Gross set/rutuam

[9] Goleman, D. (1995). Emotional Intelligence. New York: Bantam Books. Kimonis, E., Frick, P., Munoz, L. \&Aucoin, K. (2007). Can a laboratory measure of emotional processing enhance the statistical prediction of aggression and delinquency in detained adolescents with callous-unemotional traits? Journal of Abnormal Child Psychology, 35, 773-785. doi: 10.1007/s10802-007-9136-1

[10] Goleman, D. (1995). Emotional Intelligence. New York: Bantam

[11] Johnston, A. W. (2003). A correlational study of emotional intelligence and aggression in adolescents. Masters Abstracts International, 42(02), 368.
[12] Liau, A.K., Liau, W.L., Teoh, B.S. \&Liau, T.L.( 2003). The case for emotional literacy: the influence of emotional intelligence on problem behaviours in Malaysian secondary school students. Journal of Moral Education, 32(1):51-66.

[13] Lopes, P. N., Salovey, P. \& Straus, R. (2003).Emotional intelligence, personality and the perceived quality of social relationships. Personality and Individual Differences, 35(3), 641-659.

[14] Mayer, J. D. \&Salovey, P. (1993). The intelligence of emotional intelligence. Intelligence, 17, 433-442.

[15] Myers, B. (1996).Raising Responsible Teenagers. London: Jessica Kingsley

[16] Mayer, J. D. and P. Salovet. (1997). "What is Emotional Intelligence?" (pp. 3-27), in P. Salovey and D.J. Sluyter (eds.), Emotional Development and Emotional Intelligence: Educational Implications. New York: Basic Books.

[17] Petrides, K. V., Sangareau, Y. Furnham, A. \& Frederickson, N. (2006). Trait emotional intelligence and children's peer relations at School. Social Development, 15(3), 537-547

[18] Patrick, C. J., Bradely, M. M. \& Lang, P. J. (1993). Emotion in the criminal psychopath: Startle reflex modulation. Journal of Abnormal Psychology, 102, 82-92.

[19] Plug, C., Louw, D.A., Gouws, L.A., and Meyer, W.F. (1997). VerklarendeSielkundigeWoordeboek[Explanatory Psychological Dictionary]. Johannesburg: Heinemann

[20] Parker, J.D.A. (Ed), Summerfeldt, L.J., Hogan, M.J. \&Majeski, S.A. (2004). Emotional intelligence and academic success: Examining the transition from high school to university. Personality and Individual Differences, 36:163-172.

[21] Pipher, M. (1994). Reviving Ophelia. Saving Lives of Adolescent Girls. New York: Ballantine

[22] Qualter, P., Gardner, K. J. \&Whiteley, H.E. (2007). Emotional intelligence: Review of research and educational implications. Pastoral Care, 11-20.

[23] Steinberg, L. \&Cauffman, E. (1996). Maturity of judgment in adolescence: Psychosocial factors in adolescent decision making. Law and Human Behaviour, 20, 249-272.

[24] Steinberg, L. \& Scott, E. S. (2003). Less guilty by reason of adolescence:

[25] Singh, S. \&Saini, S. (2007). Emotional Intelligence, need structure, unconscious conflicts and coronary heart disease. Indian Journal of Applied Psychology, 44, 43-49.

[26] Slotboom, A., Hendriks, J., \&Verbruggen, J. (2011). Contrasting adolescent female and male sexual aggression: A self-report study on prevalence and predictors of 23 sexual aggressions. Journal of Sexual Aggression, 17(1), 1533. doi:10.1080/13552600.2010.544413

[27] Salovey, P. \& Mayer, J. D. (1990) Emotional intelligence. Imagination, Cognition and Personality, 9(3), 185-211

[28] Sadock, J.B., \&Sadock, V.A. (2003). Synopsis of Psychiatry: Behavioural Sciences / Clinical Psychiatry. New York: Lippincott Williams \& Wilkins.

[29] Trinidad, D. R., Unger, J. B., Chou, C. P., Azen, S. P. \& Johnson, C. A. (2004). Emotional intelligence and smoking risk factors in adolescents: Interactions on smoking intentions. Journal of Adolescent Health, 34(1), 46-55. 
[30] Winters, J., Clift, R. J. W. \& Dutton, D. G. (2004). An exploratory study of emotional Intelligence and domestic abuse. Journal of Family Violence, 19, 255-267.

[31] Zeman, J., Shipman, K. \&Suveg, C. (2002). Anger and sadness regulation: Predictions tointernalizing and externalizing symptoms in children. Journal of Clinical Child and Adolescent Psychology, 31, 393-398.
[32] Larry J. Siegel, Brandon C. Welsh (2007). Why Males are more aggressive than females ,Juvenile Justice; The Core: "Second Edition" 